\title{
Carburation d'alliage d'aluminium par traitement de surface par laser excimère
}

\author{
F. Fariaut, C. Boulmer-Leborgne, C. Langlade ${ }^{1}$ et T. Sauvage ${ }^{2}$ \\ GREMI, UMR 6606 du CNRS, Université d'Orléans, BP. 6744, 45067 Orléans cedex 2, France \\ 1 IFOS, École Centrale de Lyon, 36 avenue de Collongue, BP. 163, 69131 Ecully cedex, France \\ ${ }^{2}$ CERI, CNRS, $3 A$ rue de la Férollerie, 45071 Orléans cedex 2, France
}

\begin{abstract}
Résumé : Les alliages légers de type aluminium sont très prisés dans l'industrie automobile pour leur légèreté, mais leur mauvais comportement en usure et en frottement les rend inutilisables pour certaines applications. Pour améliorer ce comportement, une solution est de faire une couche très dure en surface, un traitement par laser à excimère de l'alliage en présence d'un gaz contenant du carbone permet une carburation de cet alliage en surface sur quelques microns d'épaisseur.

Pour ce traitement on utilise un laser à excimère $\operatorname{KrF}(\lambda=248 \mathrm{~nm}, \tau=25 \mathrm{~ns})$ dont le faisceau est homogénéisé et focalisé sur l'échantillon à traiter. L'échantillon est placé dans une enceinte à vide remplie d'un gaz carboné $\left(\mathrm{CH}_{4}\right.$ ou $\mathrm{C}_{3} \mathrm{H}_{6}$ ) à une pression de 1 bar ; pour le traitement l'échantillon peut être déplacé perpendiculairement au faisceau en X et en Y. L'ensemble laser-déplacement est piloté par ordinateur. Ce procédé permet un traitement de la surface localisé aux endroits de fortes sollicitations mécaniques et non polluant.
\end{abstract}

\section{INTRODUCTION}

Pour durcir la surface d'un alliage il a été montré que le traitement par laser en atmosphère d'azote permet la formation de nitrures sur plusieurs microns sur de l'acier [1,2], et sur de l'aluminium [3-5]. Le même traitement sur l'alliage $A l \mathrm{Ai}_{7 q_{\mathrm{f}}} \mathrm{Mg}_{0.3 \%}$ en atmosphère de gaz carboné $\left(\mathrm{C}_{\mathrm{p}} \mathrm{H}_{\mathrm{m}}\right)$ doit permettre l'incorporation de carbone et la formation de carbures. Les paramètres influençant le traitement sont la fluence laser $\left(\mathrm{J} / \mathrm{cm}^{2}\right)$, le nombre de tirs laser par unité de surface et le gaz de traitement. Les premiers essais ont été faits avec du méthane $\left(\mathrm{CH}_{4}\right)$, la comparaison des quantités de carbone incorporé par rapport aux quantités d'azote incorporées avec une atmosphère $\mathrm{N}_{2}$ a montré qu'il fallait environ trois fois plus de tirs laser pour incorporer autant de carbone que d'azote pour des conditions de traitement identiques.

\section{CARbURATION}

Des traitements d'alliage d'aluminium ont été réalisés pour un nombre de tirs laser par unité de surface allant de 100 à 1200 , deux gaz de traitement $\left(\mathrm{CH}_{4}\right.$ et $\left.\mathrm{C}_{3} \mathrm{H}_{6}\right)$ et une fluence laser de $2.4 \mathrm{~J} / \mathrm{cm}^{2}$ supérieure à
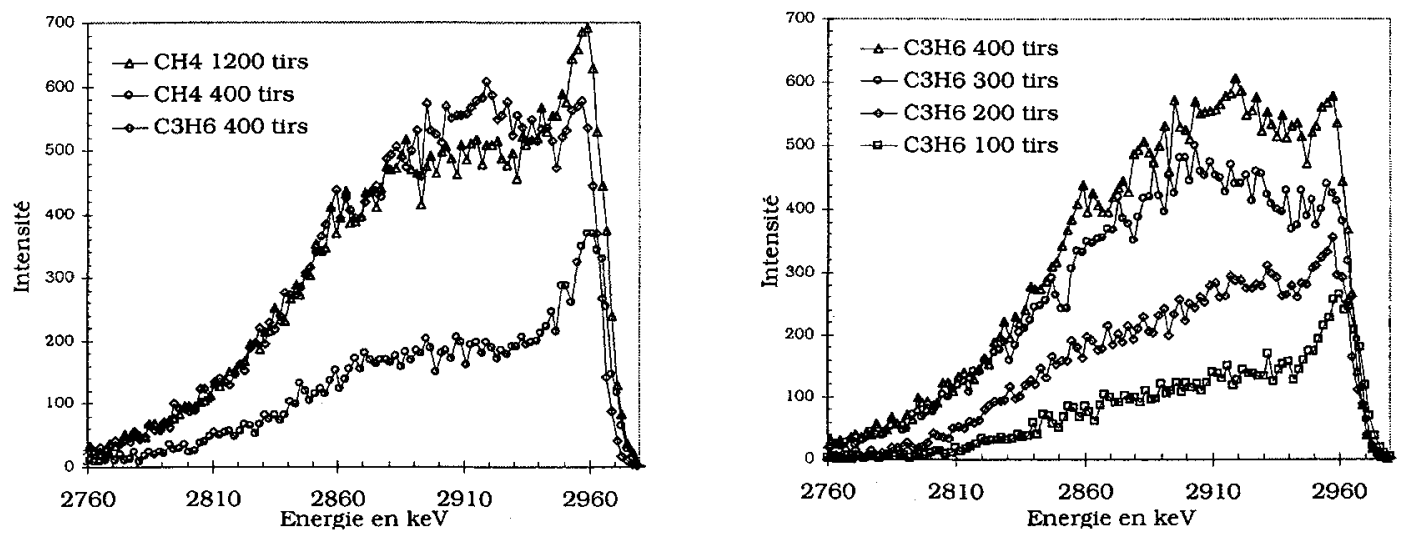

Figure 1: Spectres NRA de la réaction ${ }^{12} \mathrm{C}\left(\mathrm{d}, \mathrm{p}_{0}\right)^{13} \mathrm{C}$ pour les différentes conditions de traitement. 
l'énergie seuil d'obtention d'un plasma en surface $2 \mathrm{~J} / \mathrm{cm}^{2}$ indispensable à la formation d'une couche de carbure.

La quantité de carbone incorporée [C] est analysée par NRA (faisceau de deuton de $1 \mathrm{MeV}$, réaction $\left.{ }^{12} \mathrm{C}\left(\mathrm{d}, \mathrm{p}_{0}\right){ }^{13} \mathrm{C}\right)$. Sur la figure 1 les énergies les plus fortes en abscisse sont représentatives de la surface de l'échantillon et les énergies les plus basses de la profondeur. Les intensités en ordonnées sont proportionnelles à $[\mathrm{C}]$. D'après ces résultats il est clair qu'on incorpore environ trois fois plus de carbone avec un traitement sous atmosphère de $\mathrm{C}_{3} \mathrm{H}_{6}$ qu'en atmosphère de $\mathrm{CH}_{4}$. De même, on voit que le nombre de tirs laser permet d'augmenter [C]. On peut voir que de bons résultats peuvent êtres obtenus avec le propylène $\left(\mathrm{C}_{3} \mathrm{H}_{6}\right)$ et 400 tirs laser. Des analyses par rayons $\mathrm{X}$ en incidence rasante ont montré la présence de phases cristallines de carbure d'aluminium $\mathrm{Al}_{4} \mathrm{C}_{3}$ sur des profondeurs supérieures à $1 \mu \mathrm{m}$.

\section{ESSAIS TRIBOLOGIQUES}

Pour vérifier l'amélioration du comportement mécanique de la surface, des tests de fretting plan-cylindre ont étés réalisés. Ces essais consistent à faire frotter un cylindre d'acier $100 \mathrm{C} 6 \mathrm{de} 20 \mathrm{~mm}$ de diamètre sur un échantillon traité. On mesure le coefficient de frottement (force tangentielle / force normale appliquée) en fonction du nombre de cycles de frottement, l'amplitude de frottement est de $10 \mu \mathrm{m}$.

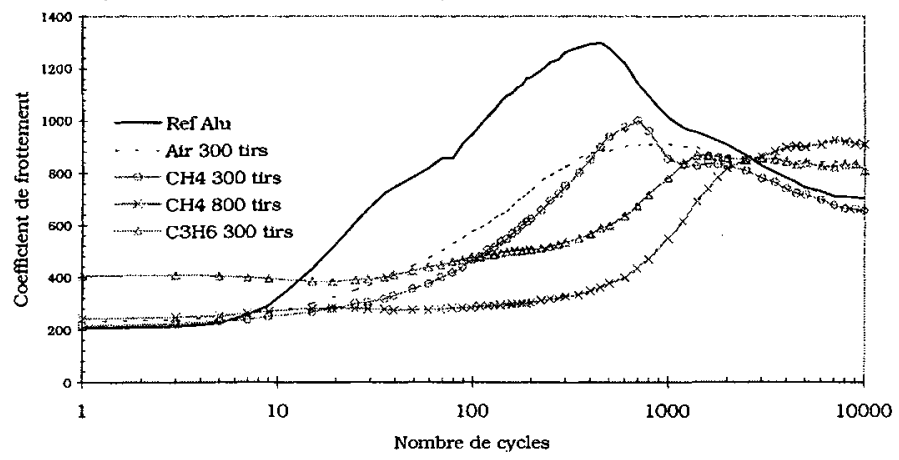

Figure 2: Evolution du coefficient de frottement pour différentes conditions de traitement.

L'évolution du coefficient de frottement donne une information qualitative sur la stabilité de la couche en surface, un coefficient de frottement qui reste constant indique dans notre cas une usure modérée de la couche. On voit sur la figure 2 que dans le cas de l'alliage de référence (ref. Alu) le coefficient de frottement dérive rapidement, cela correspond à une évolution de la morphologie et des propriétés de la surface conduisant à sa dégradation. Pour les échantillons traités avec 300 tirs laser en atmosphère de méthane ou d'air, la dégradation est comparable, le traitement a durci la surface mais la couche superficielle est rapidement endommagée. Les meilleurs résultats sont obtenus avec les traitements sous $\mathrm{C}_{3} \mathrm{H}_{6} 300$ tirs et $\mathrm{CH}_{4} 800$ tirs, la couche résiste à environ 800 cycles de frottements.

\section{CONCLUSION}

Cette étude montre l'intérêt du procédé de carburation pour l'amélioration des propriétés tribologiques d'un alliage d'aluminium et l'importance du choix du gaz ambiant permettant cette carburation.

Ce travail est soutenu par PREDIT n ${ }^{\circ} 97$ T 0293.

\section{Références}

1. P. Schaaf, M.Han - Hyperfine Interactions 0 (2000) 1-4.

2. F.Landry, K.-P. Lieb, P.Schaaf - Journal of Applied Physics 86 (1) (1999) 168.

3. E. Sicard, C. Boulmer-Leborgne, C. Andreazza-Vignolle, P. Andreazza, C. Langlade, B. Vannes - Surface and Coating Technology 100-101 (1998) 440-444.

4. E. Sicard, C. Boulmer-Leborgne, T. Sauvage - Applied Surface Science 127-129 (1998) 726-730.

5. E. Sicard, C. Boulmer-Leborgne, M. Fraisnais - J.Phys. IV France 9 (1999) 157-160. 